\title{
POLE mutations improve the prognosis of endometrial cancer via regulating cellular metabolism through AMF/AMFR signal transduction
}

\author{
Yiran Li', Yiding Bian', Kai Wang ${ }^{2}$ and Xiao-Ping Wan ${ }^{1 *}$ (D
}

\begin{abstract}
Background: The morbidity and mortality of endometrial tumors, a common type of malignant cancer in women, have increased in recent years. POLE encodes the DNA polymerase $\varepsilon$, which is responsible for the leading strand DNA replication. Somatic mutations of POLE have been acknowledged in numerous cancers, resulting in the accumulation of DNA errors, leading to ultra-mutated tumors. Mutations in the exonuclease domain of POLE have been reported to improve progression-free survival in endometrial cancer. However, the potential relationship and underlying mechanism between POLE mutations and the prognosis of endometrial cancer patients remains unclear.

Methods: The whole exome sequencing data, RNA sequencing data, and clinical information were obtained from the TCGA database and employed for the analyses in this study. The detailed mutational information was analyzed using whole exome sequencing data and the mutated genes were shown with OncoPlot. The survival curves and cox proportional hazards regression analysis were used to accessed patient prognosis, the association of clinical characteristics and prognosis. Differentially expressed genes were analyzed by the edgeR R/Bioconductor package, then the GSEA Pre-ranked tool was used for Gene Set Enrichment Analysis (GSEA) to estimate the function of genes. Expression values were clustered using hierarchical clustering with Euclidean distance and ward linkage by the dendextend R package.
\end{abstract}

Results: POLE mutational status was proven to be an independent prognostic factor for endometrial cancer patients. Patients with somatic POLE mutations presented a favorable prognosis. POLE mutations regulated glycolysis and cytokine secretion, affecting cell metabolism and immune response. Autocrine motility factor (AMF)/PGI and AMFR/gp78 exhibited higher expression levels in POLE mutant patients. The comprehensive high expressions of AMFR/gp78 and low expression of POLE were associated with the favorable prognosis of endometrial cancer patients.

Conclusions: This study showed the POLE mutations a vital factor in endometrial cancer patients, leading to a higher expression of AMF/PGI and AMFR/gp78. These results suggested comprehensive consideration of the POLE mutations, expression of AMF/PGI and AMFR/gp78 may provide a more feasible and effective approach for the treatment of endometrial cancer, which might improve the prognosis.

Keywords: Endometrial cancer, Polymerase $\varepsilon$ (POLE) mutation, Glucose metabolism, Autocrine motility factor (AMF), Prognosis

\footnotetext{
* Correspondence: wanxiaoping62@126.com

${ }^{1}$ Department of Gynecology, Shanghai First Maternity and Infant Hospital,

Tongji University School of Medicine, 2699 Gaoke West Road, Shanghai,

China

Full list of author information is available at the end of the article
}

(c) The Author(s). 2019 Open Access This article is distributed under the terms of the Creative Commons Attribution 4.0 International License (http://creativecommons.org/licenses/by/4.0/), which permits unrestricted use, distribution, and reproduction in any medium, provided you give appropriate credit to the original author(s) and the source, provide a link to the Creative Commons license, and indicate if changes were made. The Creative Commons Public Domain Dedication waiver (http://creativecommons.org/publicdomain/zero/1.0/) applies to the data made available in this article, unless otherwise stated. 


\section{Background}

Endometrial cancer (EC), arising from the lining of the uterus, is one of the most common malignancy among women in developed countries. The incidence and mortality of endometrial cancer have risen in the United States in recent years, with estimated 74,200 new cases and 151,30 deaths in 2017, and about 76,470 new cases and 15,520 deaths in 2018 [1-4]. Although most patients present low-grade, early-stage diseases with favorable prognosis, the high-grade group accounts for a large proportion of deaths in endometrial cancer patients [5]. In addition, the mutation frequency profile was also related to different ethnicities and tumor grades in endometrial cancer patients [6]. PIK3CA, ARID1A, TP53, PIK3R1, and MUC16 are the most frequent mutations in endometrial cancer patients, and all of them have been reported to be associated with prognosis. PIK3CA, ARID1A, and MUC16 mutations were correlated with favorable prognosis, whereas TP53 and PIK3R1 mutations were correlated with poor prognosis $[6,7]$.

All cancers are caused by alterations in the DNA sequence including somatic mutations of the genomes in normal cells. DNA polymerases (Pols) are important enzymes involved in DNA replication and repair in vivo. According to the similarity of the protein sequence, Pols are categorized into six families: A, B, C, D, X and Y. Pols $\alpha, \delta$, and $\varepsilon$ are three DNA polymerases essential during DNA replication in eukaryotes [8]. POLE (p261) encodes the DNA polymerase $\varepsilon$, which is a heterotetramer (p261, p59, p17, p12) [9] and responsible for the leading strand DNA replication $[10,11]$. POLE contains the proof-reading exonuclease domain to ensure low mutation rates in replicating cells [12]. Early studies demonstrated that POLE mutations were observed in colorectal cancer, pancreatic cancer, ovarian cancer, ultra-mutated giant cell high-grade glioma, and endometrial cancer [13-17]. Germline mutations in the exonuclease domains of POLE were associated with a familial predisposition and increased risk of colorectal cancer and giant cell glioblastoma [18-20]. Somatic mutations in POLE have also been reported in these cancers [21]. These mutations lead to an accumulation of DNA errors during the replication process, resulting in ultra-mutated tumors associated with microsatellite stability [22].

Different forms of genomic instability in cancers are known to be associated with prognosis [23]. The somatic POLE mutations can lead to a genomically, histologically and clinically distinct subgroup of high-grade gliomas that are associated with a longer progression-free survival [16]. In endometrial cancer, somatic POLE proofreading mutations were found in about $7 \% \mathrm{ECs}$, and the results have shown a tight association with high histologic grade [24]. Mutations in the exonuclease domain of POLE have been confirmed to be significantly associated with favorable prognosis and improved progressionfree survival in endometrial cancer [25-27]. These results all suggest that POLE exonuclease domain mutations can serve as an important prognostic molecular marker associated with excellent outcomes and guide the management of endometrial cancer patients. The role of POLE and POLE mutants in carcinogenesis and the progression of endometrial carcinoma is not fully clear. In this study, we integrated whole exome sequencing data, patient clinical information, and the RNA sequencing data of the Uterine Corpus Endometrial Carcinoma (UCEC) project in the TCGA database for combinational analysis to elucidate the possible mechanism of POLE mutations on endometrial cancer.

\section{Methods}

\section{TCGA data downloading}

In this study, 530 endometrial cancer patients with whole exome sequencing data, 528 patients with clinical and survival information, and 526 patients with RNA expression data in the TCGA-UCEC project were downloaded from the TCGA database. In brief, the Mutation Annotation Format (MAF) data generated by the mutect2 algorithm from whole exome sequencing data, RNA sequencing raw counts (HTSeq-Counts) data, RNA sequencing FPKM data (HTSeq-FPKM), clinical and survival information were downloaded from the TCGA database through the TCGAbiolinks R/Bioconductor package [28].

\section{Analyses and visualization of somatic mutations}

The detailed mutational information was extracted from the MAF file by the Maftools R/Bioconductor package [29]. Next, the plotmafSummary function was used to plot the summary of the MAF file for displaying the number of variant type and variant classification. The oncoplot function was used to plot the OncoPlot of the top 10 mutated genes and POLE. The lollipopPlot of POLE was plotted through the lollipopPlot function [29].

\section{Prognostic analyses}

We used the Kaplan-Meier method and compared it with the log-rank test to depict the survival curves. Cox proportional hazards regression analysis was used for univariate and multivariate analyses to explore the association of clinical characteristics, POLE mutational status, and patient prognosis.

\section{Differentially expressed genes and enrichment analysis}

The differentially expressed genes (DEGs) between the mutant and the wild type POLE endometrial cancer samples were analyzed by the edgeR $\mathrm{R} /$ Bioconductor 
package [30]. Gene ontology (GO) and the Kyoto Encyclopedia of Genes and Genomes (KEGG) were used for the enrichment analysis of the function and signaling pathway of the DEGs through the hypergeometric algorithm. The value of $p<0.05$ was considered significantly enriched.

\section{Gene set enrichment analysis}

Gene Set Enrichment Analysis (GSEA) was performed through the GSEA Pre-ranked tool in the GSEA software (http://www.broadinstitute.org/gsea/). The value of $\log _{2}(\mathrm{FC})$ calculated by the edgeR package was used as the ranking metric. DEGs and all the other genes were both analyzed by GSEA. We used the C5 collection, which contains gene sets annotated by GO terms, and the C2 canonical pathway sub-collection contains 1329 gene sets that are annotated pathways mainly from the KEGG, BIOCARTA and REACTOME databases in the analysis.

\section{Hierarchical clustering}

RNA sequencing FPKM data of 526 endometrial cancer samples in the TCGA database were used to assess the gene expression levels. Unsupervised hierarchical clustering was used to distinguish groups based on the expression pattern of target genes. Expression values of these genes in the 526 samples (where rows indicate the identity of the genes, columns indicate the identity of the patients) were clustered using hierarchical clustering with Euclidean distance and ward linkage by the dendextend $\mathrm{R}$ package [31] and heatmap.2 function in the gplots R package.

\section{Results}

\section{POLE frequently mutates in endometrial Cancer}

In this study, 530 endometrial cancer patients containing the whole exome sequencing data in the TCGA-UCEC project were included for subsequent analysis.

Somatic alterations on a multiscale were identified in the exome of each endometrial cancer case including single-nucleotide polymorphisms (SNPs), short insertions (INS), and short deletions (DEL). In all 530 exome sequencing data of endometrial cancer samples, 886,371 somatic mutations were identified based on consensus calls from the mutect2 mutation-detection algorithm. $73.21 \%$ of endometrial cancer patients (Number $=388)$ contained less than 500 somatic mutations, 16.98\% (Number $=90$ ) contained 500-2000 somatic mutations, $2.64 \%$ (Number $=14$ ) contained $2000-5000$ somatic mutations and $7.17 \%($ Number $=38)$ contained more than 2000 somatic mutations (Fig. 1a). The survival curve of endometrial cancer patients based on the number of mutations (mutation number $>500$ or $<500$ ) showed that the endometrial cancer patients had an improved prognosis when they held more mutations $(P=0.00033)$ (Fig. 1b). The patients with POLE somatic mutations had a higher tumor mutational burden compared with patients without POLE mutations $(P=$ 1.26e-76) (Fig. 1c). These results suggest that POLE mutations may be an important prognostic factor in patients with endometrial cancer. In these somatic mutations, the major type of variants were SNPs (Number = $821,967)$, which was mainly the missense mutation (Additional file 1: Table S1). Silent, intron, UTR, 5' flank, 3' flank, splice region, and other synonymous SNPs were filtered and only non-synonymous variants were selected for further analysis. In the nonsynonymous variants, most variants were SNPs and the major mutation was missense mutation (Additional file 1: Figures S1A, B). The top 10 mutated genes in endometrial cancer were: PTEN (57\%), PIK3CA (48\%), TTN (44\%), ARID1A (43\%), TP53 (36\%), MUC16 (30\%), PIK3R1 (30\%), KMT2D (27\%), CTCF (24\%), and CSMD3 (24\%). In addition, 15\% of endometrial cancer patients had POLE somatic mutations as well, where more than $90 \%$ of POLE mutations were missense mutations (Fig. 1d).

POLE was the 123rd most frequently mutated gene in endometrial cancer with a mutational frequency of 80 in the 530 tumor samples (15\%) (Fig. 1b, Additional file 1: Table S2). We identified 144 mutations in the POLE gene including 140 SNPs, 3 INS, and 1 DEL. In detail, the 144 mutations included 131 missense mutations, 7 nonsense mutations, 3 Frame_Shift_Ins, 1 Frame_Shift_ Del, and 2 Splice_Site mutations (Additional file 1: Figures S1C, D). Most SNPs in POLE were substitutions between guanine and adenine (G/A) (Additional file 1: Figure S1E). Except for the 2 Splice_Site mutations, 142 somatic mutations occurred in exon regions containing 39 exons and more than 33\% mutations occurred in exon9 (17.6\%) and exon13 (16.2\%) (Fig. 1e). The remaining $66.2 \%$ mutations were relatively evenly distributed in the other 37 exons (all exons contained less than 5\% mutations). Most of the POLE mutations were missense mutations and these mutations were equally distributed from the $\mathrm{N}$-terminal to the $\mathrm{C}$-terminal of the POLE protein (Fig. 1f).

\section{POLE mutations are associated with improved prognosis of endometrial Cancer patients}

A total of 528 endometrial cancer patients with clinical and survival information, including histological type, histologic grade, clinical stage, age at initial pathologic diagnosis, hypertension, history of neoadjuvant treatment, and diabetes in the TCGA-UCEC project, were used in this series. The detailed clinical characteristics are listed in Table 1. To explore the relationship between the POLE mutations and the clinical characteristics, we employed 


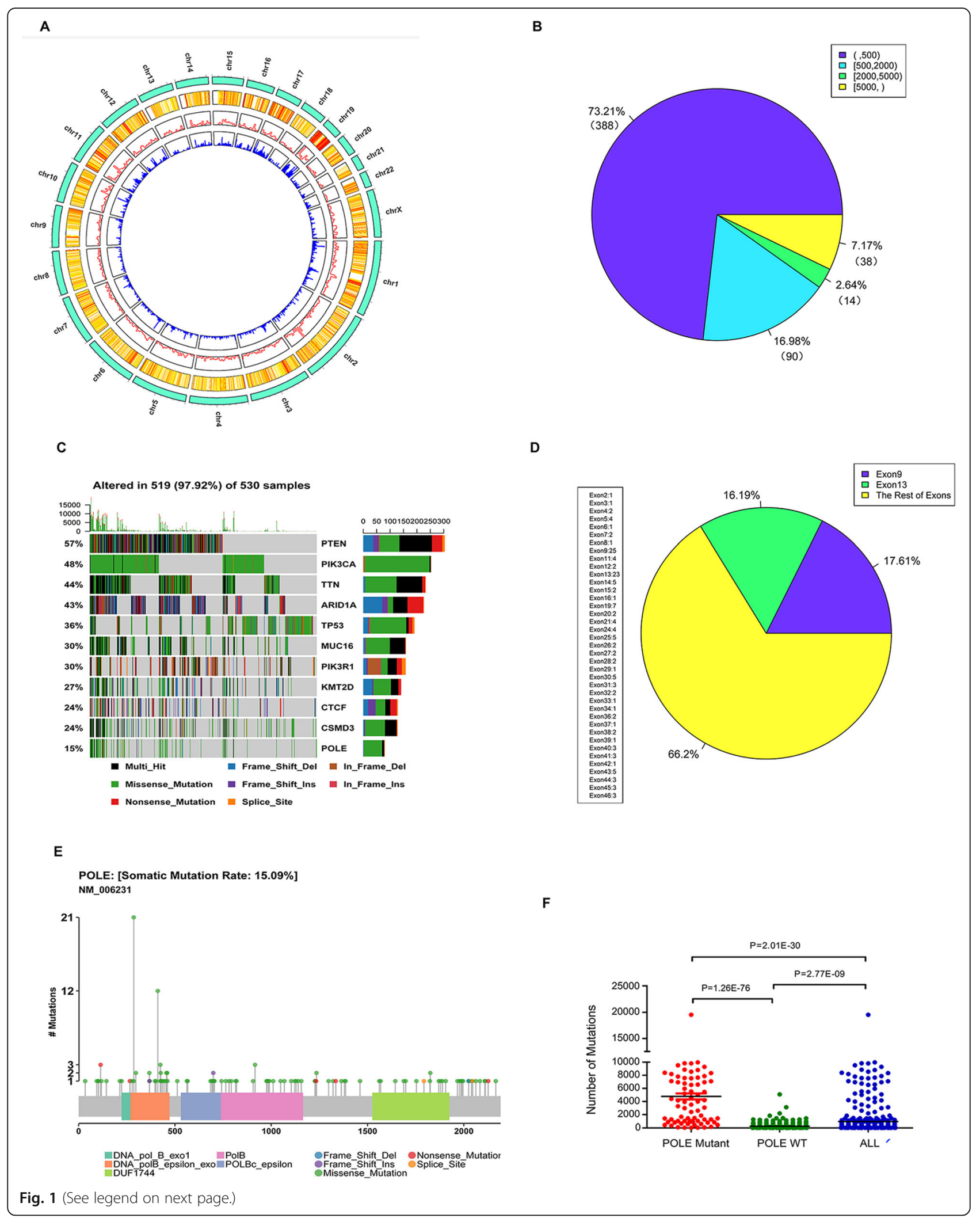


(See figure on previous page.)

Fig. 1 POLE frequently mutates in endometrial cancer. Somatic mutations were analyzed from the exome sequencing data of 530 endometrial cancer samples in the TCGA-UCEC project. a The distribution of the somatic mutation number in the endometrial cancer samples. b KaplanMeier curve and log-rank test for endometrial cancer patients based on mutation number. c The number of mutations for all patients $(N=530)$, patients with POLE mutant $(N=80)$, and patients with wild type POLE $(N=450)$. The data were compared using the wilcox.test. The bars represent the mean and SEM. d The OncoPlot of the top 10 mutated genes and POLE. The upper barplot indicates the number of mutations per patient, while the right barplot shows the number of mutations per gene. The mutation types were added as annotations at the bottom. Variants annotated as Multi_Hit are genes that mutated more than once in the same sample. e The pie chart of mutations of POLE occurring in multiple exons. $\mathbf{f}$ The lollipopPlot of POLE. The amino acid axis is labeled for domain. The mutation types were added as annotations at the bottom

the chi-square test to establish the relationship between the POLE mutations and histological type, histologic grade, clinical stage, and age at initial pathologic diagnosis. The results showed that POLE mutational status was significantly associated with the histological type $(P=$ $0.0006766)$ and age at the initial pathologic diagnosis $(P=$ $4.39 \mathrm{e}-05)$. No significant relationship was found between the POLE mutational status and the histologic grade or clinical stage $(P>0.05)$ (Additional file 1: Table S3).

Table 1 Clinical characteristics of patients with endometrial cancer

\begin{tabular}{|c|c|}
\hline Clinical characteristics & Cases (\%) \\
\hline \multicolumn{2}{|l|}{ Histological type } \\
\hline Endometrioid endometrial adenocarcinoma & $395(74.81 \%)$ \\
\hline Mixed serous and endometrioid & $22(4.17 \%)$ \\
\hline Serous endometrial adenocarcinoma & $111(21.02 \%)$ \\
\hline \multicolumn{2}{|l|}{ Histologic grade } \\
\hline G1 & $97(18.37 \%)$ \\
\hline $\mathrm{G} 2+\mathrm{G} 3$ & $431(81.63 \%)$ \\
\hline \multicolumn{2}{|l|}{ Clinical stage } \\
\hline $1+\|$ & $381(72.16 \%)$ \\
\hline III + IV & $147(27.84 \%)$ \\
\hline \multicolumn{2}{|l|}{ Age at initial pathologic diagnosis } \\
\hline$<60$ & $174(32.95 \%)$ \\
\hline$\geq 60$ & $352(66.67 \%)$ \\
\hline$N A^{a}$ & $2(0.38 \%)$ \\
\hline \multicolumn{2}{|l|}{ Hypertension } \\
\hline YES & $260(49.24 \%)$ \\
\hline NO & 178(33.71\%) \\
\hline NA & $90(17.05 \%)$ \\
\hline \multicolumn{2}{|l|}{ History of neo adjuvant treatment } \\
\hline YES & $2(0.38 \%)$ \\
\hline NO & $526(99.26 \%)$ \\
\hline \multicolumn{2}{|l|}{ Diabetes } \\
\hline YES & $108(20.45 \%)$ \\
\hline NO & $300(56.82 \%)$ \\
\hline NA & $120(22.73 \%)$ \\
\hline
\end{tabular}

${ }^{\mathrm{a}}$ NA Not available
The correlation between patient survival and histologic grade, clinical stage and POLE mutations, respectively, was analyzed by the Kaplan-Meier method and log-rank test. The results showed all three features correlate with patient survival with a $P$ value less than 0.05 (Figs. 2a-c). Next, we conducted a Cox proportional hazards regression analysis to further investigate the correlation between patient overall survival and the four clinical features, POLE mutations, respectively. The $P$ values of all five features were less than 0.05 in the univariate analysis, whereas, in the multivariate analysis, clinical stage $(P=2.50 \mathrm{E}-08)$, histologic grade $(P=0.00378)$, and POLE $(P=0.00194)$ mutational status were shown to be independent prognostic factors in endometrial cancer patients (Table 2). The results showed that endometrial cancer patients with low histologic grade (G1), early clinical stage (I+ II), and POLE mutations had favorable prognosis (Figs. 2a-c). Compared with the clinical stage, which showed the smallest $P$ value in the Cox proportional hazards regression analysis and Kaplan-Meier curve in all three independent prognostic factors, histologic grade and POLE mutations were good stratified factors because it did not intersect in their survival curves.

Previous results suggest that POLE mutational status was associated with tumor mutational burden in endometrial cancers [32] consistent with our finding (Fig. 1c). To a certain extent, it is also possible that the hypermutated status might affect the prognosis of endometrial cancer patients. In order to increase the accuracy of the impact from $P O L E$ mutations and minimize the interference of hypermutated status on patient prognosis, we excluded hypermutated phenotypes (more than 500 mutations per sample) and found that the total tumor mutational burden decreased significantly. The survival curve of endometrial cancer patients showed that the exclusion of patients with hypermutated phenotypes altered the effects of POLE mutations on patient prognosis, hence the POLE mutations were no longer significantly associated with improved prognosis (Additional file 1: 

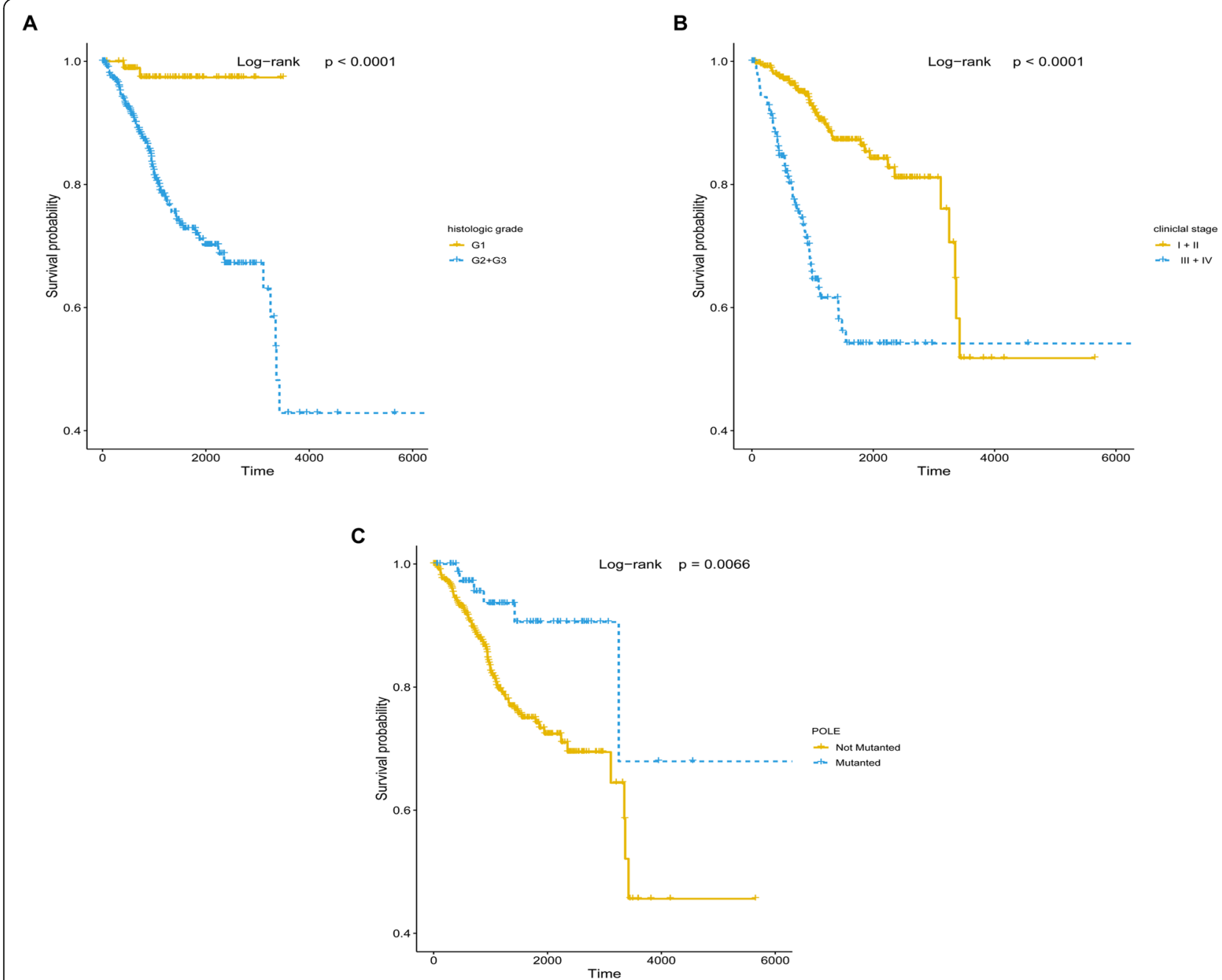

Fig. 2 Histologic grade, clinical stage, and POLE mutational status are independent prognostic factors for endometrial cancer patients. a KaplanMeier curve and log-rank test for endometrial cancer patients based on histologic grade classification. b Kaplan-Meier curve and log-rank test for endometrial cancer patients based on clinical stage classification. c Kaplan-Meier curve and log-rank test for endometrial cancer patients based on POLE mutational status classification

Figure S2). The variation in the relationship between $P O L E$ mutations and prognosis may be due to the limited sample size of the POLE mutant after removing the hypermutated phenotypes (Number $=10)$. The effect of mutational burden could not be considered in this study due to the limited number of samples.

\section{POLE mutations are involved in cellular glucose} metabolism

Endometrial cancer patients with POLE mutations had favorable prognoses, indicating that mutant POLE may play an important role in the development and progression of endometrial cancer. To further investigate the

Table 2 Univariate and multivariate Cox regression analysis in endometrial cancer patients

\begin{tabular}{|c|c|c|c|c|c|c|}
\hline \multirow[t]{2}{*}{ Clinical characteristics } & \multicolumn{3}{|c|}{ Univariate Cox regression analysis } & \multicolumn{3}{|c|}{ Multivariate Cox regression analysis } \\
\hline & $\exp ($ coef) & $P$ & $95 \% \mathrm{Cl}$ & $\exp ($ coef) & $P$ & $95 \% \mathrm{Cl}$ \\
\hline Histological type & 1.661 & $6.26 \mathrm{e}-06$ & 1.333 to 2.070 & 1.064 & 0.62231 & 0.831 to 1.363 \\
\hline Histologic grade (G1 vs. G2 + G3) & 1.116 & 0.00076 & 1.047 to 1.189 & 1.1 & 0.00378 & 1.031 to 1.173 \\
\hline Clinical stage $(I+||$ vs. III + IV) & 1.063 & $5.38 \mathrm{e}-10$ & 1.043 to 1.084 & 1.062 & $2.50 \mathrm{e}-08$ & 1.040 to 1.085 \\
\hline $\begin{array}{l}\text { Age at initial pathologic diagnosis } \\
(<60 \text { vs. } \geq 60)\end{array}$ & 1.803 & 0.0158 & 1.117 to 2.910 & 1.489 & 0.12497 & 0.895 to 2.478 \\
\hline POLE (WT vs. mutated) & 0.334 & 0.00968 & 0.146 to 0.767 & 0.256 & 0.00194 & 0.108 to 0.606 \\
\hline
\end{tabular}


role of POLE mutations in endometrial cancer, we used transcriptome sequencing data from 526 patients to analyze the global gene expression profile and the differential expression between the two groups in 80 POLE mutant patients and 446 patients with wild type POLE. A total of 1587 differentially expressed genes (DEGs) were obtained according to the cut-off criteria $(P<0.05$ and $\mathrm{FC}>1.5$ ) including 725 upregulated genes and 862 downregulated genes (Figs. 3a). To clarify the functional differences between these two groups, we conducted the gene set enrichment analysis (GSEA), which is a bioinformatics method that determines whether a set of terms shows statistically significant enrichment [33]. GSEA using Gene Ontology (GO) terms as the gene sets showed the enrichment in categories like glucose metabolic process, isomerase activity, exonuclease activity, and intramolecular transferase activity in endometrial cancer samples with POLE mutations (Figs. 3b-e). The $\mathrm{T}$ cell receptor signaling pathway and the production of molecular mediators of immune response were also significantly enriched in the GSEA results (Additional file 1: Figures S3A, B). GO analysis using the hypergeometric algorithm of 1587 DEGs was similar as those of the GSEA, which indicated that cytokine receptor activity, cytokine binding, cytokine receptor binding, chemokine receptor activity, and carbohydrate binding were enriched (Additional file 1: Figure S3C).

To further enlighten us in targeting the cellular metabolism for POLE mutations, canonical pathways mainly from the KEGG, BIOCARTA, and REACTOME databases as the gene sets were used for GSEA. The results showed that cellular glucose metabolism such as glycolysis and gluconeogenesis were significantly enriched in endometrial cancer samples with POLE mutations (Figs. 4a, b). These findings suggest that POLE mutations can regulate cellular glucose metabolism in endometrial cancer patients.

In order to further study the function of POLE mutations in cellular metabolism through glucose metabolism, we analyzed the expression levels of 20 genes in glucose metabolism and 18 genes in glycolysis in endometrial cancer samples between the POLE mutant and the wild type. Endometrial cancer samples were reclassified into two clusters based on the gene expression patterns of these two pathways according to hierarchical clustering. We also examined the mutation rate of POLE according to hierarchical clustering. In glucose metabolism, cluster I had a higher gene expression pattern and exhibited a higher rate of POLE mutations; yet cluster II had a lower expression pattern and lower rate of POLE mutations (Fig. 4c). Therefore, the elevation of glucose metabolism significantly correlated with a higher rate of POLE mutations. Similarly, two main groups were also observed in glycolysis. Cluster I had a higher gene expression pattern and exhibited a higher rate of $P O L E$ mutations, while cluster II had a lower expression pattern and a lower rate of POLE mutations (Fig. 4d). Therefore, the elevation of cellular glucose metabolism significantly correlated with a higher rate of POLE mutations. These results suggest that the favorable prognosis of POLE mutants may be partly due to the regulation of cell metabolism.

\section{POLE mutations regulate AMF/PGI-AMFR/gp78 expression and patient favorable prognosis}

To a certain extent, the results of this study have demonstrated that $P O L E$ mutations may impact endometrial cancer patient prognosis by regulating cellular glucose metabolism. However, the key molecules involved in the regulation of glucose metabolism by the POLE mutations are still unclear. To further target the possible core genes, we analyzed the process of glucose metabolism in detail. Phosphoglucose isomerase (PGI) is a glycolytic enzyme involved in the gluconeogenesis-glycolysis pathways. It is an extracellular cytokine as well, under the name of autocrine motility factor (AMF). Therefore, AMF/PGI plays a dual role as a phosphoglucose isomerase that catalyzes the interconversion of glucose-6phosphate and fructose-6-phosphatein glycometabolism when it is effective as a cytokine [34-36]. Transcriptomic profiling analysis of 587 endometrial cancer and normal samples revealed differences in the expression of AMF/PGI between endometrial cancer patients and normal samples. In endometrial cancer patients, the expression of AMF/PGI (fold change $=2.51, P=6.3 \mathrm{e}-31$ ) increased significantly (Fig. 5a). This result suggests that AMF/PGI may play an important role in endometrial cancer. On the other hand, the expression of AMF/PGI has also been detected in POLE mutant and wild type endometrial cancer patients. In endometrial cancer patients with POLE mutations, the expression of AMF/PGI was slightly higher than that in patients with wild type POLE ( $F C=1.2, P=0.00077$ ), (Fig. $5 b)$. AMFR/gp78 is a cell surface receptor for AMF/PGI, which is also located in the endoplasmic reticulum where it functions as an E3 ubiquitin ligase. Stimulation of AMFR/gp78 by its ligand AMF/PGI alters cell adhesion, proliferation and apoptosis. AMFR/gp78 expression has been reported in numerous types of human tumors [35, 37]. In POLE mutants and wild type endometrial cancer patients, the expression pattern of AMFR/gp78 was similar as that of AMF/PGI ( $F C=1.26, P=0.00002$ ) (Fig. 5c). To further confirm the correlation between POLE mutations and the expression of these two genes, we used the chisquare test to establish the relationship between the POLE mutations and the expression levels of these two genes. The results showed that POLE mutational status 
A

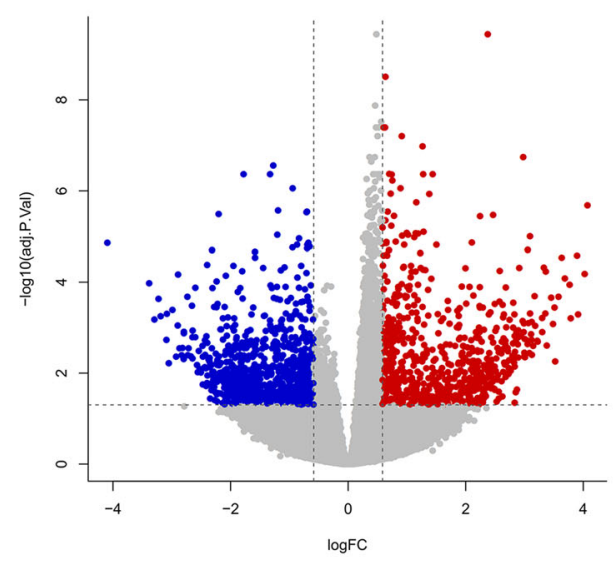

C

Enrichment plot: GO_GLUCOSE_METABOLIC_PROCESS

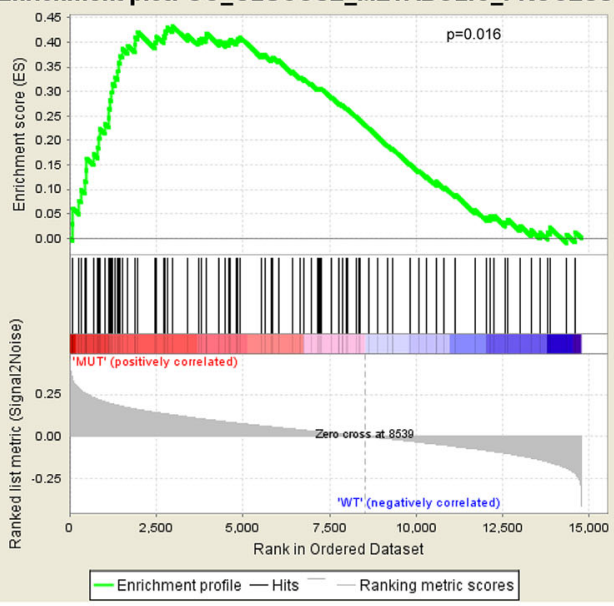

E

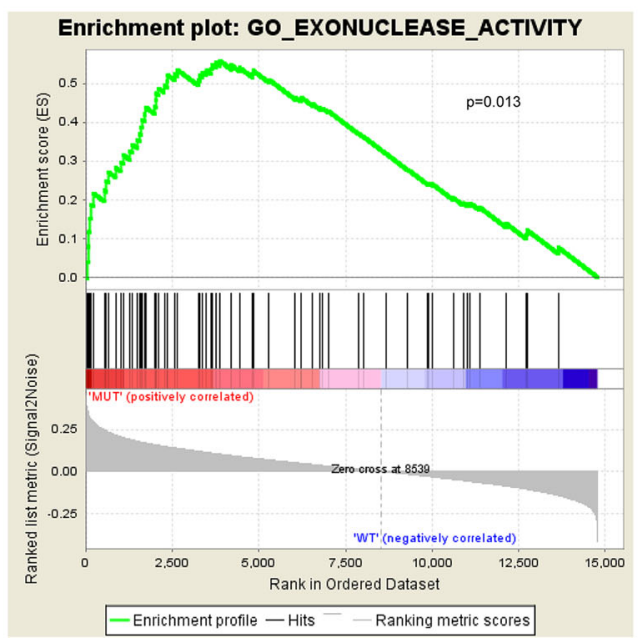

B
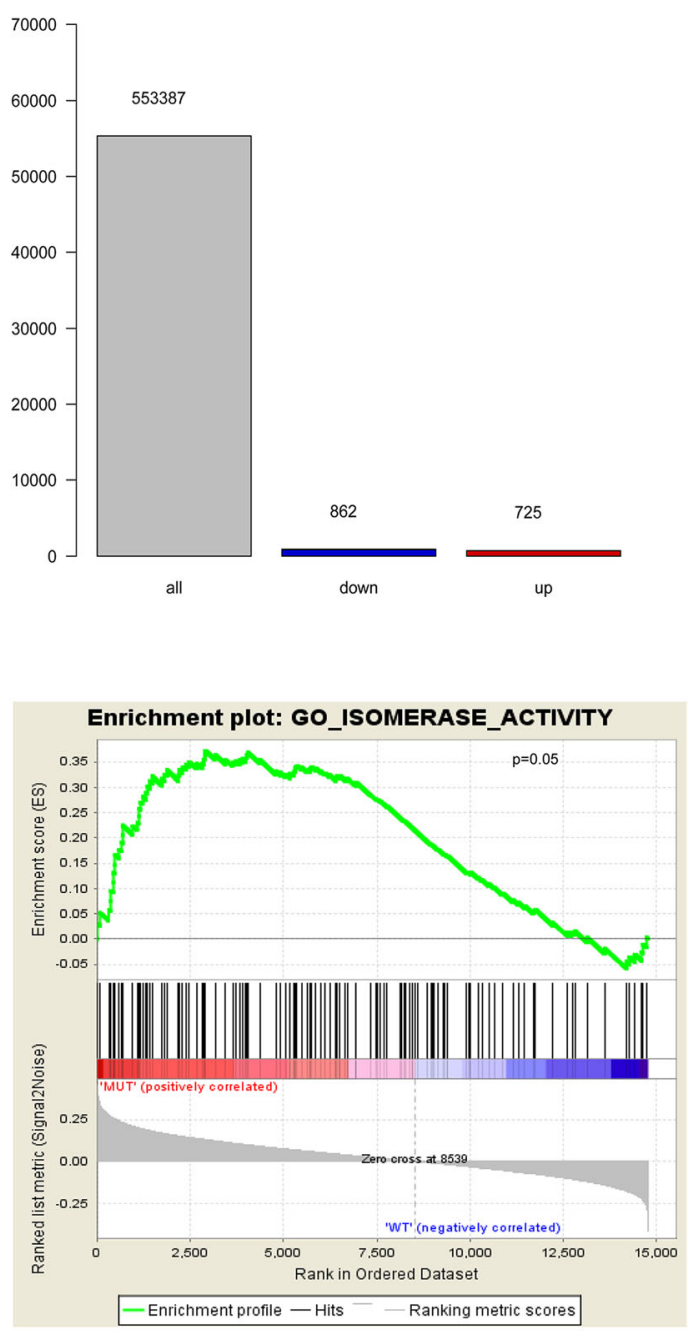

F

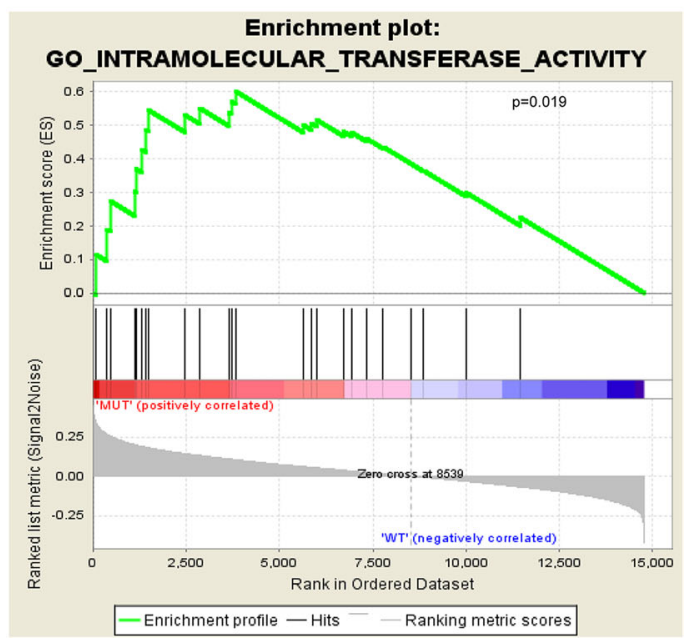

Fig. 3 (See legend on next page.) 
(See figure on previous page.)

Fig. 3 Mutated POLE is involved in glucose metabolism of endometrial cancer patients. a Volcano plot of differentially expressed genes (DEGs) between the mutant and wild type POLE endometrial cancer samples. $X$ represents the foldchange, $Y$ represents the $P$ value. Red dot represents upregulated genes while the blue dot represents downregulated genes. b-e Gene Set Enrichment Analysis (GSEA) of the global gene expression profiling between mutant and wild type POLE endometrial cancer samples. Gene sets annotated by GO terms were used in the analysis. Gene set representing glucose metabolism (c), isomerase activity (d), exonuclease activity $(\mathbf{e})$ and intramolecular transferase activity (f) are rich in POLE mutants

was significantly associated with the expression of AMF/ PGI $(P=7.041 \mathrm{e}-06)$ and AMFR/gp78 $(P=0.00017)$.

To further explore the relationship between AMF/PGI or AMFR/gp78 and prognosis of endometrial cancer patients, we analyzed the correlation between the expression levels of these two genes and patient survival by the Kaplan-Meier method and log-rank test. The patient survival curves showed no significant correlation between the expression levels of AMF/PGI or AMFR/ gp78, respectively, and the prognosis of endometrial
A

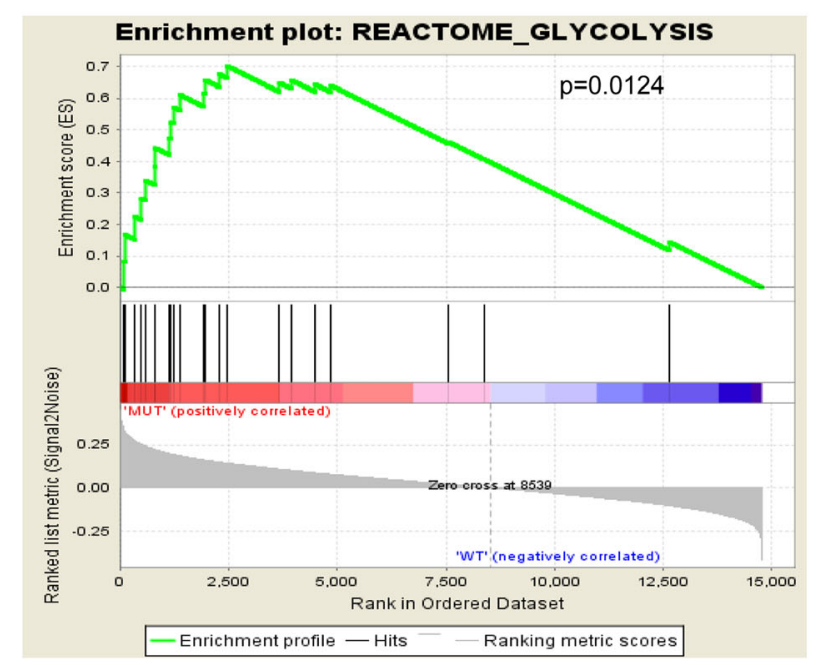

C
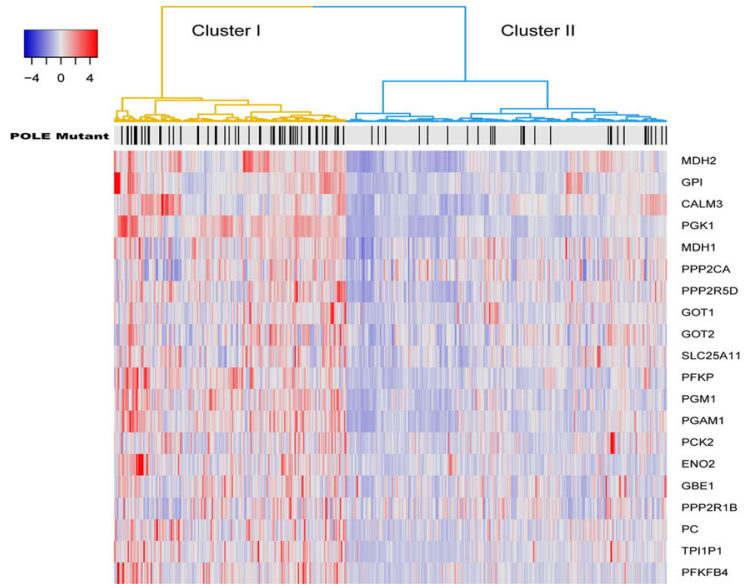

B

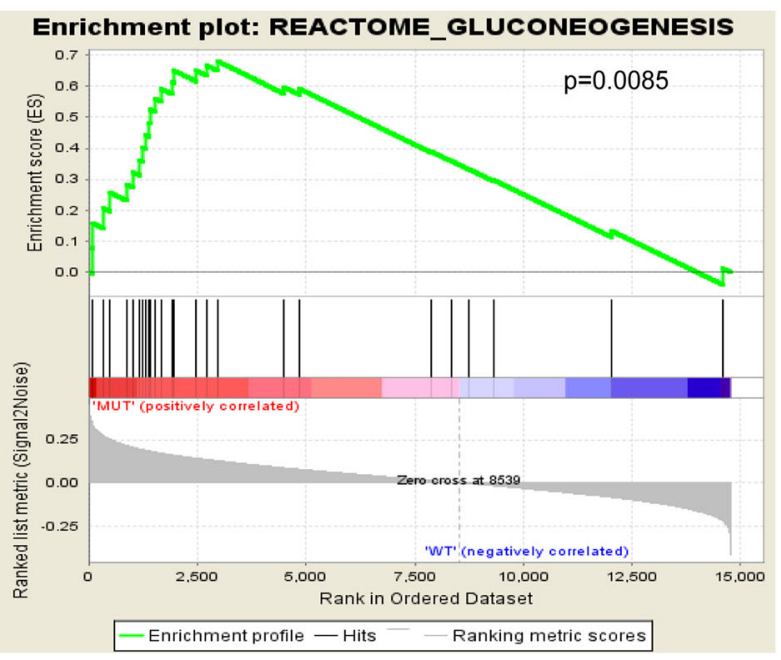

D

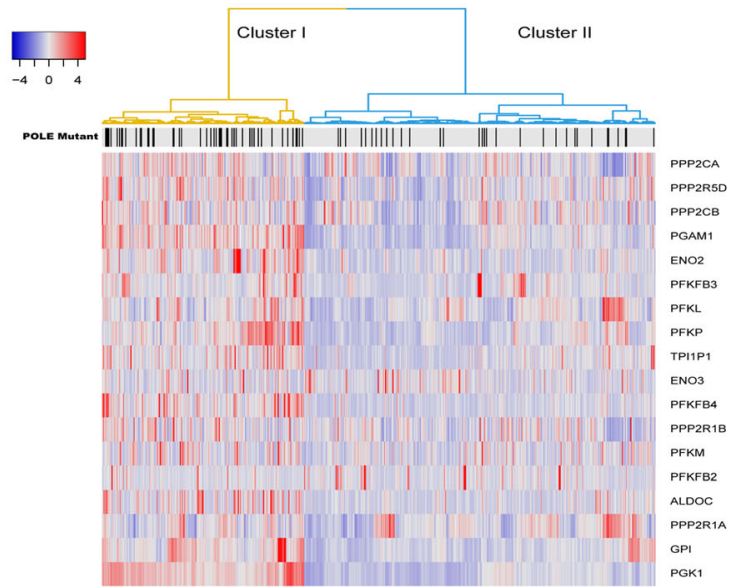

Fig. 4 Cellular glucose metabolism was enriched in POLE mutants. a, b GSEA using the canonical pathways, which are annotated pathways mainly from the KEGG, BIOCARTA and REACTOME databases, as the gene sets in the analysis. Gene sets representing glycolysis (a) and gluconeogenesis (b) were enriched in the POLE mutants. c Unsupervised hierarchical clustering with Euclidean distance and ward linkage of expression matrixes from 20 genes inglycometabolis (d) Unsupervised hierarchical clustering with Euclidean distance and ward linkage of expression matrixes from 18 genes in glycolysis. The POLE mutational status for each tumor is depicted directly on top of each column. The black vertical lines represent the samples with POLE mutations. Red represents high gene expression, and blue represents relatively low gene expression 


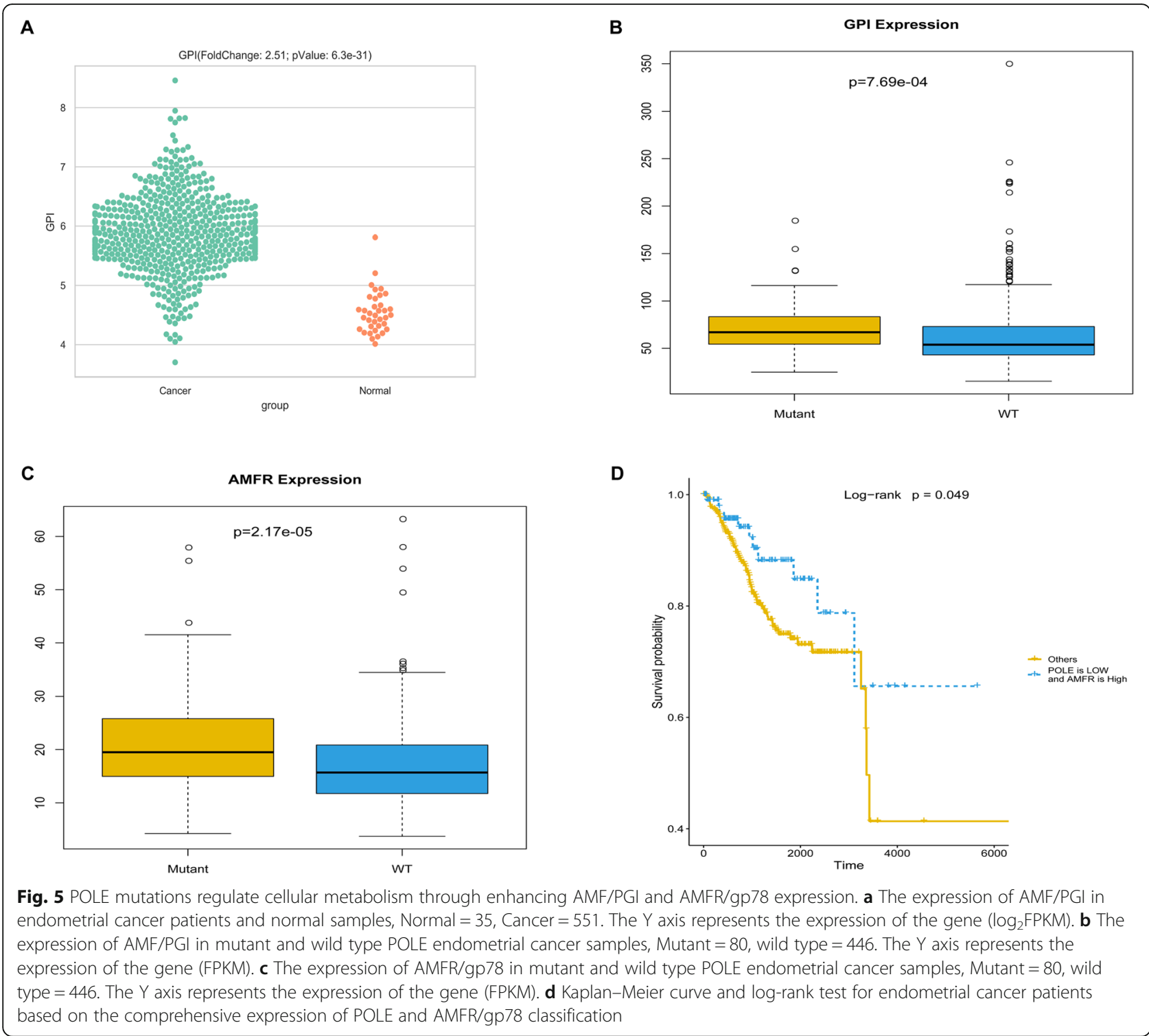

cancer patients (Additional file 1: Figures S4A, B). One interesting result was that the comprehensive expression of POLE and AMFR/gP78 was significantly correlated with patient prognosis. Patient survival curves showed high expressions of AMFR/gp78 and low expression of POLE, which were associated with the favorable prognosis of endometrial cancer patients (Fig. 5d). These results confirm the relationship between POLE mutations, AMF/PGI, AMFR/ gp78 expression, and patient prognosis, suggesting that POLE mutations may play a vital role in patient prognosis by affecting the expression and signal transduction of AMF/PGI-AMFR/gp78 as well as affecting cell metabolism.

\section{Discussion}

As a common malignancy in women, the prognosis of endometrial cancer is related to various factors such as tumor type and stage status [38]. In addition, somatic mutations have also been reported to be significantly associated with the prognosis of endometrial cancer such as MUC16 mutations and POLE exonuclease domain mutations [7, 25-27]. Although POLE exonuclease domain mutations have been reported to have a benign effect on prognosis in patients with endometrial cancer, little is known about the relationship and possible mechanism between the POLE mutational status and endometrial cancer patient prognosis. In this study, we identified the existence of somatic POLE mutations in 
80 cases of large sporadic individuals in the 530 endometrial cancer samples in the TCGA-UCEC project. We demonstrated that POLE mutations improved prognosis in endometrial cancer patients. The results of the GSEA also confirmed that cellular glucose metabolism was significantly enriched in endometrial cancer patients with POLE mutations, suggesting a possible approach to improving the prognosis of endometrial cancer through POLE mutation.

Accumulating evidence supports that driver mutations and passenger mutations both correlate with cancer progression and clinical outcomes [39, 40]. Consistently, we observed that some somatic mutations were found in many patients including PTEN, PIK3CA, TTN, etc.. Although POLE mutations were present in only 80 of 530 endometrial cancer patients, accounting for $15 \%$, many patients with POLE mutations also carried numerous passenger mutations, resulting in higher mutational burden (Fig. 1c), and these patients showed an improved prognosis (Fig. 2c). Subsequent analysis of the exclusion of these hypermutated cases altered the effects of POLE mutations on patient prognosis and no longer showed a better prognosis. As only 10 POLE mutants were included in this analysis, limited number of samples may lead to insufficient accuracy and reliability of results. Therefore, the effects of higher mutational burden on prognosis and cellular glucose metabolism should be further investigated.

Somatic mutations in POLE have been found in endometrial cancers in many studies. These reports include family studies that have confirmed the existence of novel POLE pathogenic germline variants and phenotypes of POLE-mutations in endometrial cancer (mutation analysis, clinical and lifestyle data, prognosis) [7, 32, 41, 42]. However, how do POLE mutations affect the prognosis of endometrial cancer? One novel objective of this study was to establish the possible relationship between POLE mutations and endometrial cancer prognosis. Our GSEA analyses revealed that cell glucose metabolism might play a vital role in this process. The analysis of DEGs between POLE mutant and wild type also suggests the enrichment of cell metabolism. Collectively, our data demonstrated that endometrial cancer patients with POLE mutations might achieve improved clinical prognosis by regulating cellular glycometabolis. More studies are necessary to investigate the mutant POLE in endometrial cancer therapy and how mutant POLE can be applied to benefit patients.

AMFR/gp78 is a $78 \mathrm{kDa}$ seven-transmembrane glycoprotein which stimulates with AMF/PGI and directs protein kinase $\mathrm{C}(\mathrm{PKC})$ activation and receptor phosphorylation. The levels of AMF/PGI and AMFR/ gp78 expression are associated with several clinical characteristics including metastasis and prognosis $[34,35,43-46]$. Previously, we have verified that AMF/PGI is abundant at tumor sites to affect the process of tumor growth and metastasis [47], while the expression level of AMF did not relate to the relapse-free survival in endometrial cancer patients [36]. AMF/PGI and AMFR/gp78 are key genes in this process, however there was no significant correlation between the expression of these two genes and the prognosis of endometrial cancer patients, leading to the ambiguous results. The possibility of some other gene expressions that work with AMF/PGI or AMFR/gp78 affecting patient prognosis needs to be clarified. In our results, we found that POLE expression was increased in endometrial cancer patients $(\mathrm{FC}=1.65, P=8.1 \mathrm{e}-15)$. The expression of POLE also changed slightly between the POLE mutant and the wild type ( $\mathrm{FC}=1.27, P=2.52 \mathrm{e}-5)$. We uncovered that AMF/PGI and AMFR/gp78 both had higher expression in POLE mutants and that the comprehensive low expression of POLE and high expression of AMFR/gp78 showed a positive correlation with patient survival time. POLE may have a synergistic effect on patient outcomes with AMFR/gp78, yet the mechanism needs further study.

\section{Conclusions}

In this study, we utilized whole exome sequencing data, RNA sequencing data, and clinical information in the TCGA-UCEC project downloaded from the TCGA database for comprehensive analysis. The results suggest that POLE mutations can significantly improve the prognosis of endometrial cancer patients and $15 \%$ of patients (Number $=80$ ) possessed POLE somatic mutations. The differential expressed genes between the POLE mutant and the wild type patients indicated the enrichment of glycometabolis Clustering analysis showed a higher expression of glucose metabolism genes and a higher rate of POLE mutations. Survival analysis through the Kaplan-Meier method unraveled the relationship between the comprehensive expression of POLE, AMFR/gp78 and patient prognosis.

Taken together, POLE mutations improve the prognosis of endometrial cancer via regulating cellular metabolism through AMF/AMFR signal transduction. These results may provide novel and promising approaches for the clinical treatment of endometrial cancer. More indepth research is needed to validate our findings and explore the feasibility of the developed method in clinical application. 


\section{Abbreviations}

AMF: Autocrine motility factor; DEL: Short deletions; EC: Endometrial cancer; INS: Short insertions; PGI: Phosphoglucose isomerase; POLE: DNA polymerase $\varepsilon$; SNPs: Single-nucleotide polymorphisms; TCGA: The Cancer Genome Atlas; UCEC: Uterine Corpus Endometrial Carcinoma

\section{Acknowledgements}

We thank all people contributed in this study.

\section{Authors' contributions}

Methodology, KW; software, YRL and YDB; formal analysis, YRL and YDB; data curation, YRL; writing - original draft preparation, YDB; writing-review and editing, YRL and KW and XPW; visualization, YDB; supervision, KW; project administration, XPW And all authors have read and approved the manuscript.

\section{Funding}

This study was funded by National Natural Science Foundation of China (grant number 81472427, 81672574 and 81702547), Shanghai Health System Outstanding Talents Program (grant number 2018YQ23), and the Shanghai Sailing Program (grant number 17YF1415300). The funding bodies played no role in the design of the study and collection, analysis, and interpretation of data and in writing the manuscript".

\section{Availability of data and materials}

All data used or analysed during this study are provided in TCGA database (https://cancergenome.nih.gov/), and the TCGA-UCEC project (https://portal. gdc.cancer.gov/projects/TCGA-UCEC)

\section{Ethics approval and consent to participate}

Not applicable.

\section{Consent for publication}

Not applicable.

\section{Competing interests}

The authors declare that they have no competing interests.

\section{Author details}

'Department of Gynecology, Shanghai First Maternity and Infant Hospital Tongji University School of Medicine, 2699 Gaoke West Road, Shanghai, China. ${ }^{2}$ Clinical and Translational Research Center, Shanghai First Maternity and Infant Hospital, Tongji University School of Medicine, Shanghai, China.

Received: 8 October 2019 Accepted: 8 December 2019

Published online: 21 December 2019

\section{Supplementary information}

Supplementary information accompanies this paper at https://doi.org/10. 1186/s12881-019-0936-2.

Additional file 1: Table S1. The number of mutation classification and type in 530 endometrial cancer patients. Table S2. The mutation frequency of top 124 mutated genes in 530 endometrial cancer patients. Table S3. The relationship between POLE mutations and four clinical characteristics by chi-square test. Figure S1. SNP and mutation istatistics in 530 endometrial cancer patients. Figure S2. Kaplan-Meier curve and Log-rank test for endometrial cancer patients based on POLE mutational status classification when excluded hypermutated phenotypes (more than 500 mutations per sample), $N=387$. Figure S3. (A-B) GSEA of genes between mutant and wild type POLE endometrial cancer samples. Figure S4. (A) Kaplan-Meier curve and Log-rank test for endometrial cancer patients based on expression level of AMF/GPI classification. (B) KaplanMeier curve and Log-rank test for endometrial cancer patients based on expression level of AMFR/gp78 classification.

\section{Author details}

Supplementary information accompanies this paper at https://doi.org/10. 1186/s12881-019-0936-2.'Department of Gynecology, Shanghai First
Maternity and Infant Hospital, Tongji University School of Medicine, 2699 Gaoke West Road, Shanghai, China. ${ }^{2}$ Clinical and Translational Research Center, Shanghai First Maternity and Infant Hospital, Tongji University School of Medicine, Shanghai, China.

Received: 8 October 2019 Accepted: 8 December 2019

Published online: 21 December 2019

\section{References}

1. Jemal A, Bray F, Center MM, Ferlay J, Ward E, Forman D. Global cancer statistics. CA Cancer J Clin. 2011;61(2):69-90.

2. Ferlay J, Soerjomataram I, Dikshit R, Eser S, Mathers C, Rebelo M, Parkin DM, Forman D, Bray F. Cancer incidence and mortality worldwide: sources, methods and major patterns in GLOBOCAN 2012. Int J Cancer. 2015;136(5):E359-86.

3. Siegel RL, Miller KD, Jemal A. Cancer statistics, 2017. CA Cancer J Clin. 2017; 67(1):7-30.

4. Siegel RL, Miller KD, Jemal A. Cancer statistics, 2018. CA Cancer J Clin. 2018; 68(1):7-30.

5. Creasman WT, Kohler MF, Odicino F, Maisonneuve P, Boyle P. Prognosis of papillary serous, clear cell, and grade 3 stage I carcinoma of the endometrium. Gynecol Oncol. 2004;95(3):593-6.

6. Althubiti MA. Mutation frequencies in endometrial Cancer patients of different ethnicities and tumor grades: an analytical study. Saudi J Med Med Sci. 2019:7(1):16-21.

7. $\mathrm{Hu}$ J, Sun J. MUC16 mutations improve patients' prognosis by enhancing the infiltration and antitumor immunity of cytotoxic T lymphocytes in the endometrial cancer microenvironment. Oncoimmunology. 2018;7(10): e1487914.

8. Park VS, Pursell ZF. POLE proofreading defects: contributions to mutagenesis and cancer. DNA Repair (Amst). 2019;76:50-9.

9. Bermudez VP, Farina A, Raghavan V, Tappin I, Hurwitz J. Studies on human DNA polymerase epsilon and GINS complex and their role in DNA replication. J Biol Chem. 2011;286(33):28963-77.

10. Pursell ZF, Isoz I, Lundstrom EB, Johansson E, Kunkel TA. Yeast DNA polymerase epsilon participates in leading-strand DNA replication. Science. 2007;317(5834):127-30

11. Reha-Krantz $\sqcup$. DNA polymerase proofreading: multiple roles maintain genome stability. Biochim Biophys Acta. 2010;1804(5):1049-63.

12. Heitzer $\mathrm{E}$, Tomlinson I. Replicative DNA polymerase mutations in cancer. Curr Opin Genet Dev. 2014;24:107-13.

13. Guerra J, Pinto C, Pinto D, Pinheiro M, Silva R, Peixoto A, Rocha P, Veiga I, Santos C, Santos R, et al. POLE somatic mutations in advanced colorectal cancer. Cancer Med. 2017;6(12):2966-71.

14. Guenther M, Veninga V, Kumbrink J, Haas M, Westphalen CB, Kruger S, Heinemann V, Kirchner T, Boeck S, Jung A, et al. POLE gene hotspot mutations in advanced pancreatic cancer. J Cancer Res Clin Oncol. 2018; 144(11):2161-6.

15. Ishikawa M, Nakayama K, Nakamura K, Ono R, Yamashita H, Ishibashi T, Minamoto T, lida K, Razia S, Ishikawa N, et al. High frequency of POLE mutations in synchronous endometrial and ovarian carcinoma. Hum Pathol. 2019:85:92-100

16. Erson-Omay EZ, Caglayan AO, Schultz N, Weinhold N, Omay SB, Ozduman K Koksal Y, Li J, Serin Harmanci A, Clark V et al. Somatic POLE mutations cause an ultramutated giant cell high-grade glioma subtype with better prognosis. Neuro-Oncology. 2015;17(10):1356-64.

17. Billingsley CC, Cohn DE, Mutch DG, Stephens JA, Suarez AA, Goodfellow PJ. Polymerase varepsilon (POLE) mutations in endometrial cancer: clinical outcomes and implications for lynch syndrome testing. Cancer. 2015:121(3):386-94.

18. Buchanan DD, Stewart JR, Clendenning M, Rosty C, Mahmood K, Pope BJ, Jenkins MA, Hopper JL, Southey MC, Macrae FA, et al. Risk of colorectal cancer for carriers of a germ-line mutation in POLE or POLD1. Genet Med. 2018;20(8):890-5.

19. Palles C, Cazier JB, Howarth KM, Domingo E, Jones AM, Broderick P, Kemp Z, Spain SL, Guarino E, Salguero I, et al. Germline mutations affecting the proofreading domains of POLE and POLD1 predispose to colorectal adenomas and carcinomas. Nat Genet. 2013;45(2):136-44.

20. Vande Perre P, Siegfried A, Corsini C, Bonnet D, Toulas C, Hamzaoui N, Selves J, Chipoulet E, Hoffmann JS, Uro-Coste E, et al. Germline mutation p. N363K in POLE is associated with an increased risk of colorectal cancer and giant cell glioblastoma. Familial Cancer. 2019;18(2):173-8. 
21. Briggs S, Tomlinson I. Germline and somatic polymerase epsilon and delta mutations define a new class of hypermutated colorectal and endometrial cancers. J Pathol. 2013;230(2):148-53.

22. Rayner E, van Gool IC, Palles C, Kearsey SE, Bosse T, Tomlinson I, Church DN. A panoply of errors: polymerase proofreading domain mutations in cancer. Nat Rev Cancer. 2016;16(2):71-81.

23. Walther A, Houlston R, Tomlinson I. Association between chromosomal instability and prognosis in colorectal cancer: a meta-analysis. Gut. 2008; 57(7):941-50.

24. Church DN, Briggs SE, Palles C, Domingo E, Kearsey SJ, Grimes JM, Gorman M, Martin L, Howarth KM, Hodgson SV, et al. DNA polymerase epsilon and delta exonuclease domain mutations in endometrial cancer. Hum Mol Genet. 2013;22(14):2820-8.

25. Cancer Genome Atlas Research N, Kandoth C, Schultz N, Cherniack AD, Akbani R, Liu Y, Shen H, Robertson AG, Pashtan I, Shen R, et al. Integrated genomic characterization of endometrial carcinoma. Nature. 2013;497(7447):67-73.

26. Meng B, Hoang LN, Mclntyre JB, Duggan MA, Nelson GS, Lee CH, Kobel M. POLE exonuclease domain mutation predicts long progression-free survival in grade 3 endometrioid carcinoma of the endometrium. Gynecol Oncol. 2014;134(1):15-9.

27. McConechy MK, Talhouk A, Leung S, Chiu D, Yang W, Senz J, Reha-Krantz $\sqcup$, Lee $\mathrm{CH}$, Huntsman DG, Gilks CB, et al. Endometrial carcinomas with POLE exonuclease domain mutations have a favorable prognosis. Clin Cancer Res. 2016;22(12):2865-73

28. Colaprico A, Silva TC, Olsen C, Garofano L, Cava C, Garolini D, Sabedot TS, Malta TM, Pagnotta SM, Castiglioni I, et al. TCGAbiolinks: an R/ Bioconductor package for integrative analysis of TCGA data. Nucleic Acids Res. 2016;44(8):e71.

29. Mayakonda A, Koeffler HP. Maftools: efficient analysis, visualization and summarization of MAF files from large-scale cohort based cancer studies. BioRxiv. 2016:052662

30. Robinson MD, McCarthy DJ, Smyth GK. edgeR: a Bioconductor package for differential expression analysis of digital gene expression data. Bioinformatics. 2010;26(1):139-40.

31. Galili T. Dendextend: an R package for visualizing, adjusting and comparing trees of hierarchical clustering. Bioinformatics. 2015;31(22):3718-20.

32. Veneris JT, Lee EK, Goebel EA, Nucci MR, Lindeman N, Horowitz NS, Lee L, Raut CP, Crotzer D, Matulonis U, et al. Diagnosis and management of a recurrent polymerase-epsilon (POLE)-mutated endometrial cancer. Gynecol Oncol. 2019;153(3):471-8.

33. Subramanian A, Tamayo P, Mootha VK, Mukherjee S, Ebert BL, Gillette MA, Paulovich A, Pomeroy SL, Golub TR, Lander ES, et al. Gene set enrichment analysis: a knowledge-based approach for interpreting genome-wide expression profiles. Proc Natl Acad Sci U S A. 2005;102(43):15545-50.

34. Watanabe $H$, Takehana K, Date M, Shinozaki T, Raz A. Tumor cell autocrine motility factor is the neuroleukin/phosphohexose isomerase polypeptide. Cancer Res. 1996;56(13):2960-3.

35. Fairbank M, St-Pierre $P$, Nabi IR. The complex biology of autocrine motility factor/phosphoglucose isomerase (AMF/PGI) and its receptor, the gp78/ AMFR E3 ubiquitin ligase. Mol BioSyst. 2009;5(8):793-801.

36. Li Y, Jia Y, Bian Y, Tong H, Qu J, Wang K, Wan XP. Autocrine motility factor promotes endometrial cancer progression by targeting GPER-1. Cell Commun Signal. 2019;17(1):22.

37. Chiu CG, St-Pierre P, Nabi IR, Wiseman SM. Autocrine motility factor receptor: a clinical review. Expert Rev Anticancer Ther. 2008;8(2):207-17.

38. Steiner E, Eicher O, Sagemuller J, Schmidt M, Pilch H, Tanner B, Hengstler JG, Hofmann M, Knapstein PG. Multivariate independent prognostic factors in endometrial carcinoma: a clinicopathologic study in 181 patients: 10 years experience at the Department of Obstetrics and Gynecology of the Mainz University. Int J Gynecol Cancer. 2003;13(2):197-203.

39. McFarland CD, Korolev KS, Kryukov GV, Sunyaev SR, Mirny LA. Impact of deleterious passenger mutations on cancer progression. Proc Natl Acad Sci U S A. 2013;110(8):2910-5.

40. McFarland CD, Yaglom JA, Wojtkowiak JW, Scott JG, Morse DL, Sherman MY, Mirny LA. The damaging effect of passenger mutations on Cancer progression. Cancer Res. 2017;77(18):4763-72.

41. Castellsague E, Li R, Aligue R, Gonzalez S, Sanz J, Martin E, Velasco A, Capella G, Stewart CJR, Vidal A, et al. Novel POLE pathogenic germline variant in a family with multiple primary tumors results in distinct mutational signatures. Hum Mutat. 2019:40(1):36-41.
42. Imboden S, Nastic D, Ghaderi M, Rydberg F, Rau TT, Mueller MD, Epstein E, Carlson JW. Phenotype of POLE-mutated endometrial cancer. PLoS One. 2019;14(3):e0214318.

43. Ma YT, Xing XF, Dong B, Cheng XJ, Guo T, Du H, Wen XZ, Ji JF. Higher autocrine motility factor/glucose-6-phosphate isomerase expression is associated with tumorigenesis and poorer prognosis in gastric cancer. Cancer Manag Res. 2018;10:4969-80.

44. Funasaka T, Raz A. The role of autocrine motility factor in tumor and tumor microenvironment. Cancer Metastasis Rev. 2007;26(3-4):725-35.

45. Tsutsumi S, Yanagawa T, Shimura T, Kuwano H, Raz A. Autocrine motility factor signaling enhances pancreatic cancer metastasis. Clin Cancer Res. 2004;10(22):7775-84.

46. Kho DH, Zhang T, Balan V, Wang Y, Ha SW, Xie Y, Raz A. Autocrine motility factor modulates EGF-mediated invasion signaling. Cancer Res. 2014:74(8):2229-37.

47. Li Y, Jia Y, Che Q, Zhou Q, Wang K, Wan XP. AMF/PGl-mediated tumorigenesis through MAPK-ERK signaling in endometrial carcinoma. Oncotarget. 2015;6(28):26373-87.

\section{Publisher's Note}

Springer Nature remains neutral with regard to jurisdictional claims in published maps and institutional affiliations.
Ready to submit your research? Choose BMC and benefit from:

- fast, convenient online submission

- thorough peer review by experienced researchers in your field

- rapid publication on acceptance

- support for research data, including large and complex data types

- gold Open Access which fosters wider collaboration and increased citations

- maximum visibility for your research: over $100 \mathrm{M}$ website views per year

At BMC, research is always in progress.

Learn more biomedcentral.com/submissions 